\title{
Preferential Oxidation of Carbon Monoxide catalyzed by Platinum Nanoparticles in Mesoporous Silica
}

\author{
Atsushi Fukuoka, ${ }^{, \dagger}{ }^{\dagger}$ Jun-ichi Kimura, ${ }^{\ddagger}$ Tadashi Oshio, ${ }^{\ddagger}$ Yuzuru Sakamoto, ${ }^{\ddagger}$ and Masaru \\ Ichikawa $^{\dagger}$ \\ ${ }^{\dagger}$ Catalysis Research Center, Hokkaido University, N-21 W-10, Sapporo 001-0021, Japan and \\ ${ }^{\ddagger}$ Division of Chemistry, Graduate School of Science, Hokkaido University, N-10 W-8, Sapporo 060- \\ 0810, Japan \\ E-mail: fukuoka@cat.hokudai.ac.jp
}

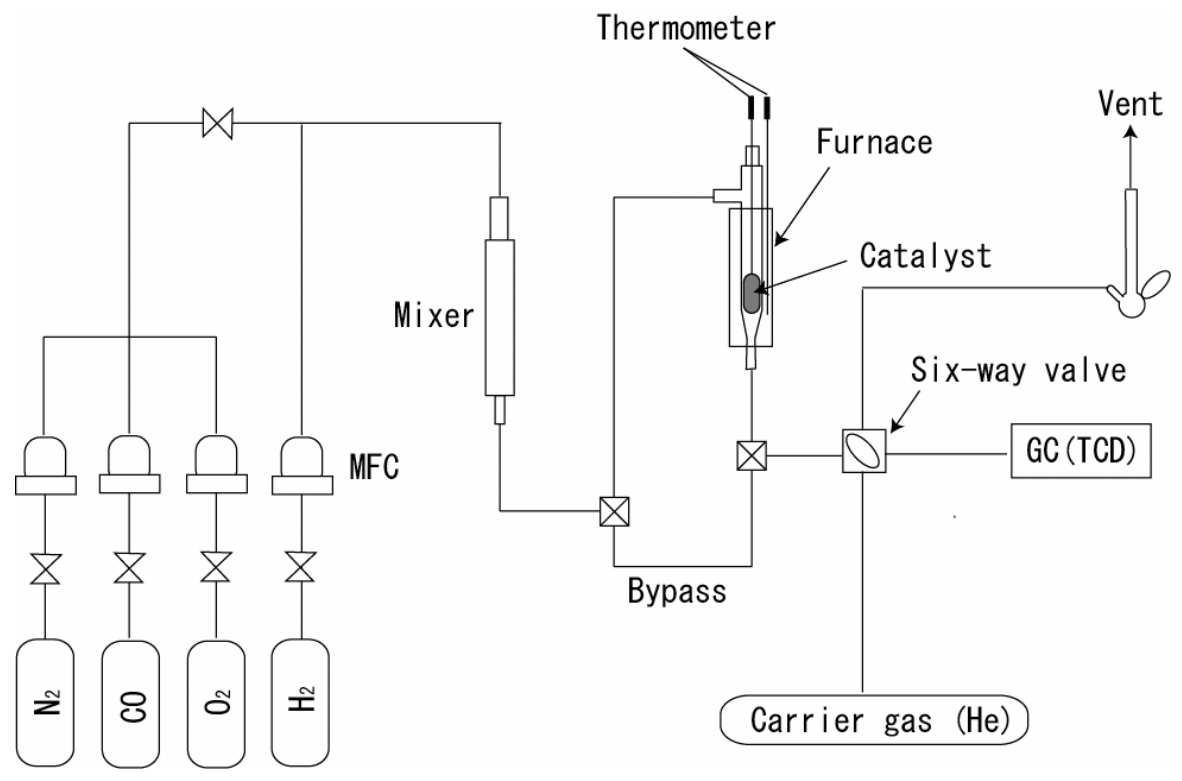

Figure S1. A plug flow reactor for PROX reaction. 


\begin{tabular}{|c|c|c|c|c|c|}
\hline Sample ID & A6221014 & & & & \\
\hline Description & FSM-16. & & & & \\
\hline \multicolumn{6}{|l|}{ Comments } \\
\hline Sample Weight & $0.0605 \mathrm{~g}$ & & & & \\
\hline Adsorbate & NITROGEN & Outgas Temp & $200.0^{\circ} \mathrm{C}$ & Operator & oshio \\
\hline Cross-Sec Area & $16.2 \AA^{2} /$ molecule & Outgas Time & $10.0 \mathrm{hrs}$ & Analysis Time & $1287.8 \mathrm{~min}$ \\
\hline NonIdeality & $6.580 \mathrm{E}-05$ & P/Po Toler & 1 & End of Run & $02 / 22 / 2006 \quad 12: 46$ \\
\hline Molecular wt & $28.0134 \mathrm{~g} / \mathrm{mol}$ & Equil Time & 5 & File Name & A6221014. RAW \\
\hline Station \# & 4 & Bath Temp. & 77.40 & PC SW Version & Pre-1.20 \\
\hline
\end{tabular}

A $\quad-O D$

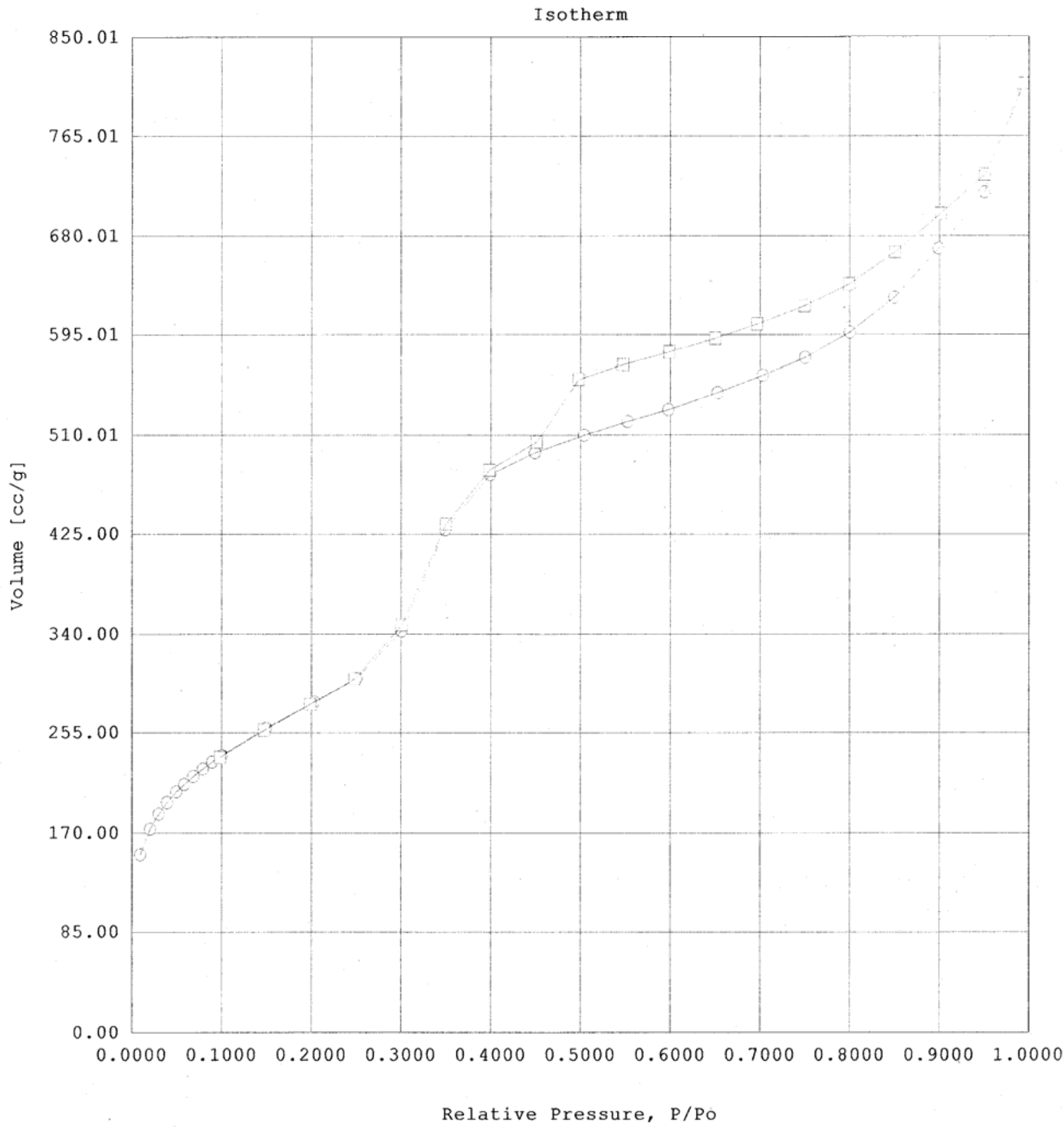

Figure S2(a). Nitrogen adsorption isotherm for FSM-16. 
Date: 03/04/2005

$$
\begin{aligned}
& P+(p) / F-5 M-16 \text { Quantachrome Corporation } \\
& 01-132 \text { Quantachrome Autosorb Automated Gas Sorption System Report } \\
& \text { Autosorb for Windows@ Version 1.27 }
\end{aligned}
$$

Sample ID A5302015

Description

Comments

Sample Weight 0.0448

Adsorbate NITROGEN

Cross-Sec Area $\quad 16.2 \AA^{2} /$ molecule

NonIdeality

$16.2 \AA^{2} /$ molecule
$6.580 \mathrm{E}-05$

Molecular wt

$28.0134 \mathrm{~g} / \mathrm{mol}$

Outgas Temp

P/Po Toler

Equil Time

1

Bath Temp.

77.40

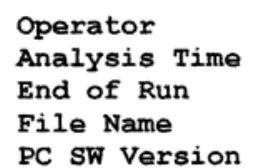

kimura

$1418.2 \mathrm{~min}$

03/03/2005 15:42

station \#

$\mathrm{A} \quad \mathrm{O}-\mathrm{D} \quad \longrightarrow$

Isotherm

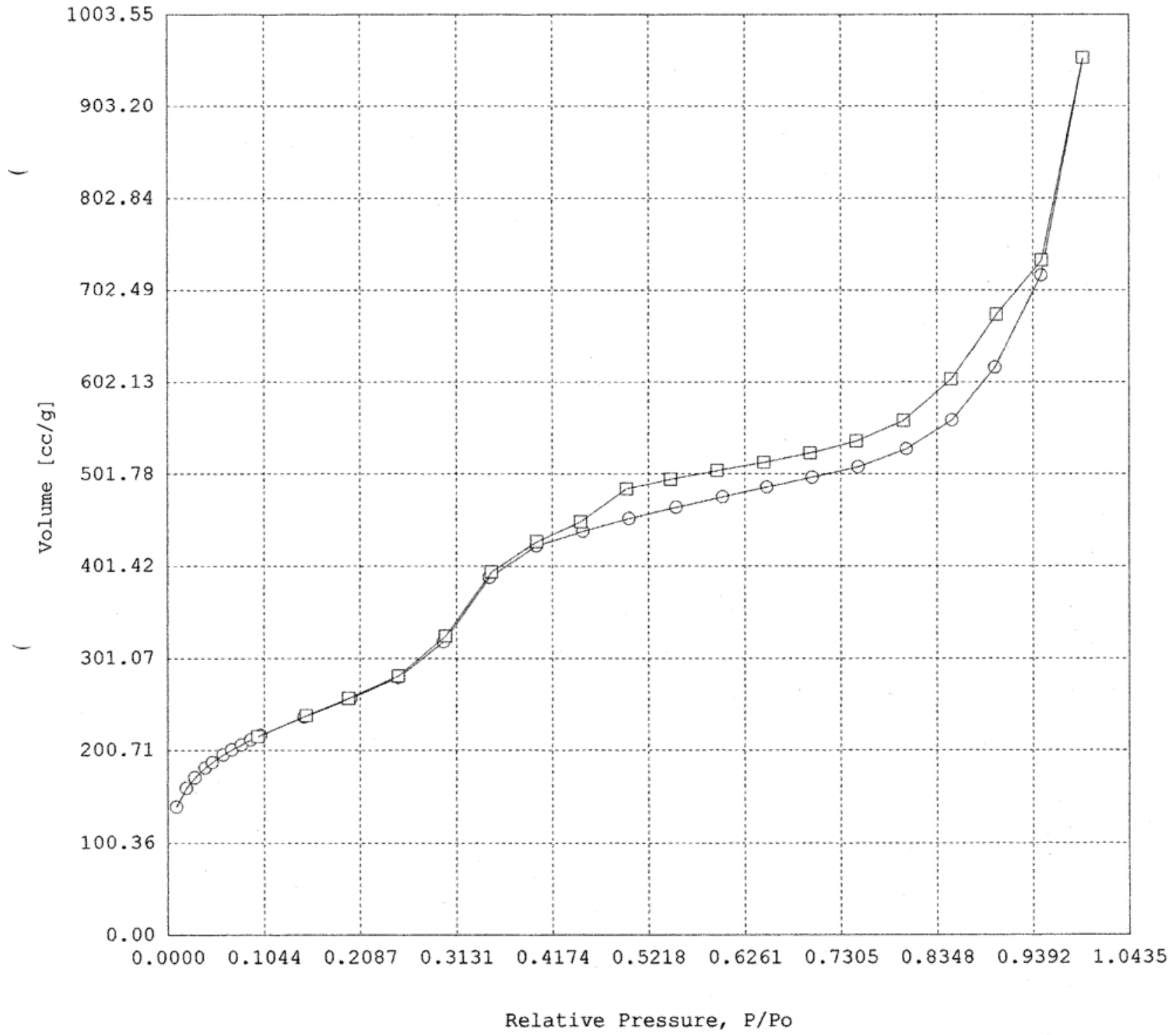

Figure S2(b). Nitrogen adsorption isotherm for Pt(p)FSM-16. 

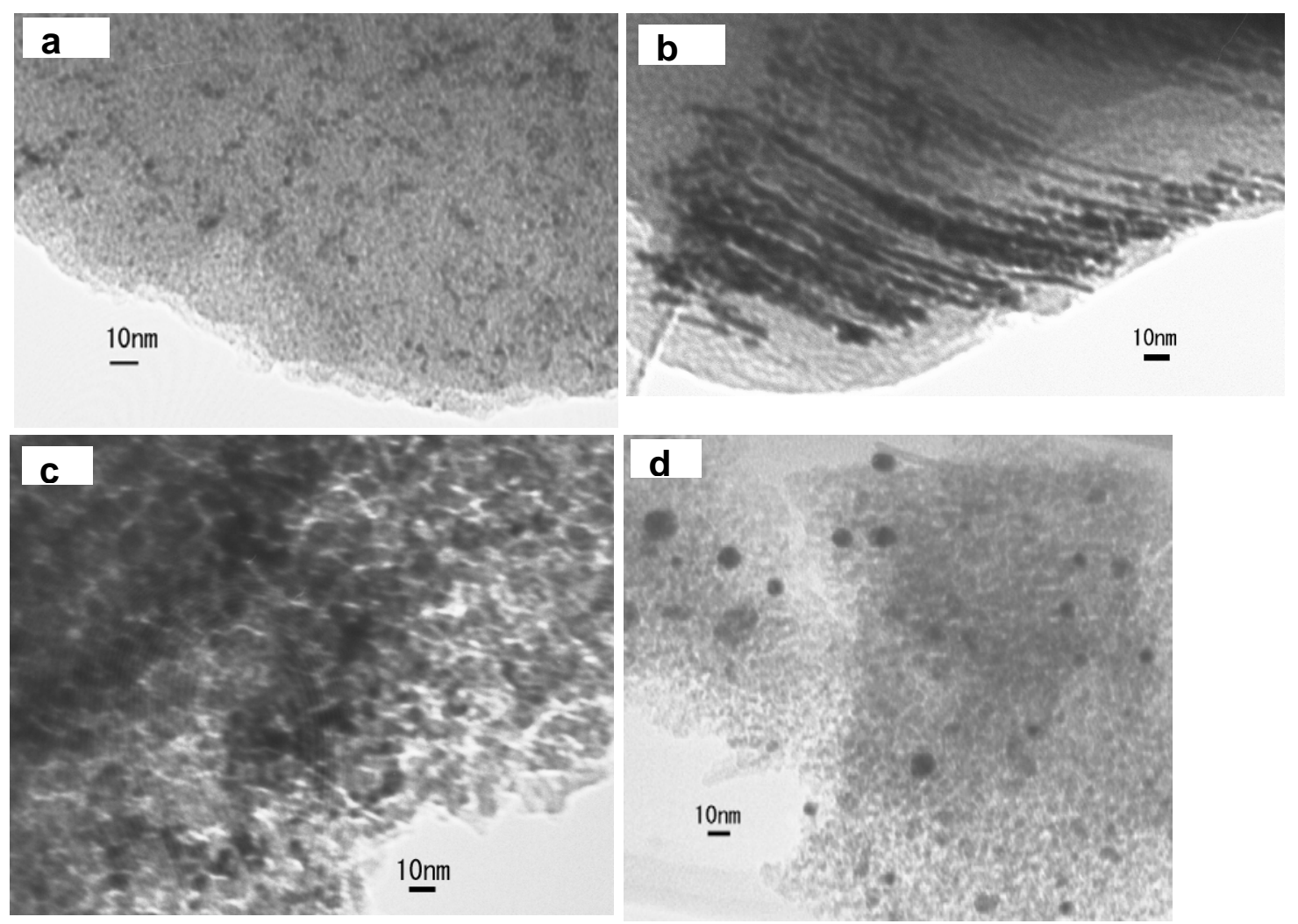

Figure S3. TEM images of (a) $\mathrm{Pt}(\mathrm{p}) / \mathrm{HMM}-1$, (b) $\mathrm{Pt}(\mathrm{w}) / \mathrm{HMM}-1$, (c) $\mathrm{Pt} / \mathrm{SiO}_{2}$ and (d) $\mathrm{Pt} / \mathrm{Al}_{2} \mathrm{O}_{3}$.
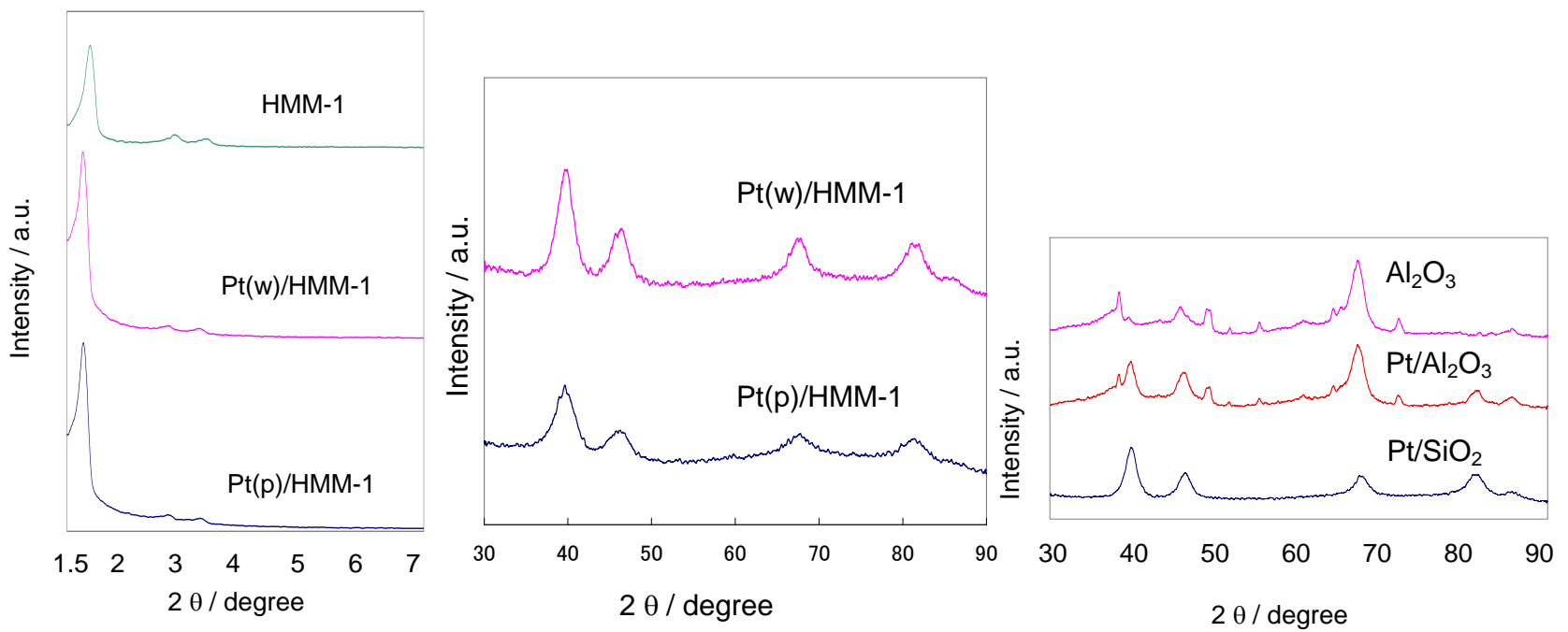

Figure S4. XRD patterns of $\mathrm{Pt}(\mathrm{p}) / \mathrm{HMM}-1, \mathrm{Pt}(\mathrm{w}) / \mathrm{FSM}-16, \mathrm{Pt} / \mathrm{Al}_{2} \mathrm{O}_{3}$ and $\mathrm{Pt} / \mathrm{SiO}_{2}$. 


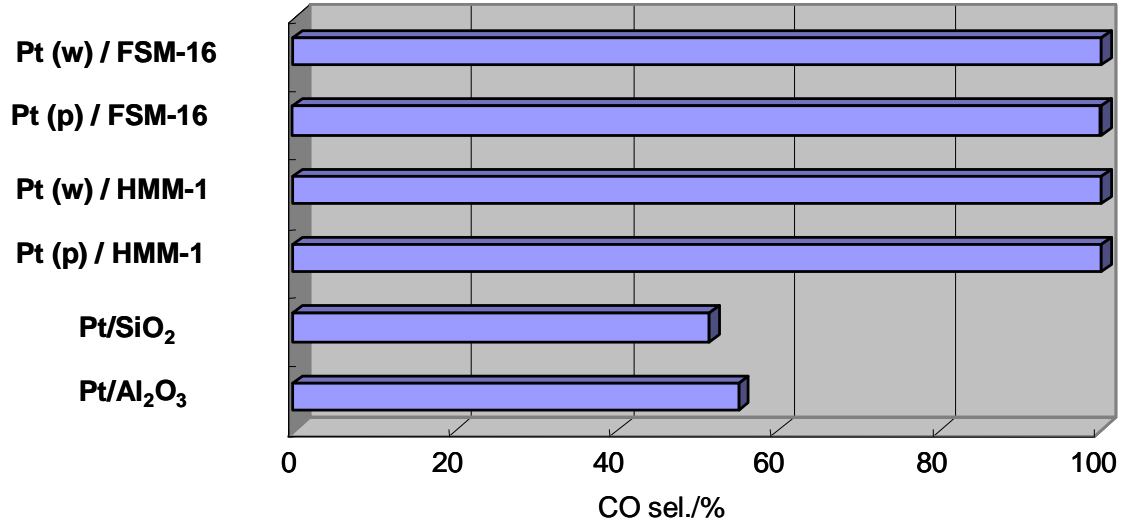

Figure S5. $\mathrm{CO}$ selectivity at 20-40\% conversion of $\mathrm{O}_{2}$. Conditions: $\mathrm{CO} 1 \%, \mathrm{O}_{2} 1 \%\left(\mathrm{O}_{2} / \mathrm{CO}=1\right), \mathrm{N}_{2} 5 \%$, $\mathrm{H}_{2}$ balance, SV $12000 \mathrm{ml} \mathrm{g}^{-1} \mathrm{~h}^{-1}, 0.1 \mathrm{MPa}, 298-423 \mathrm{~K}$.
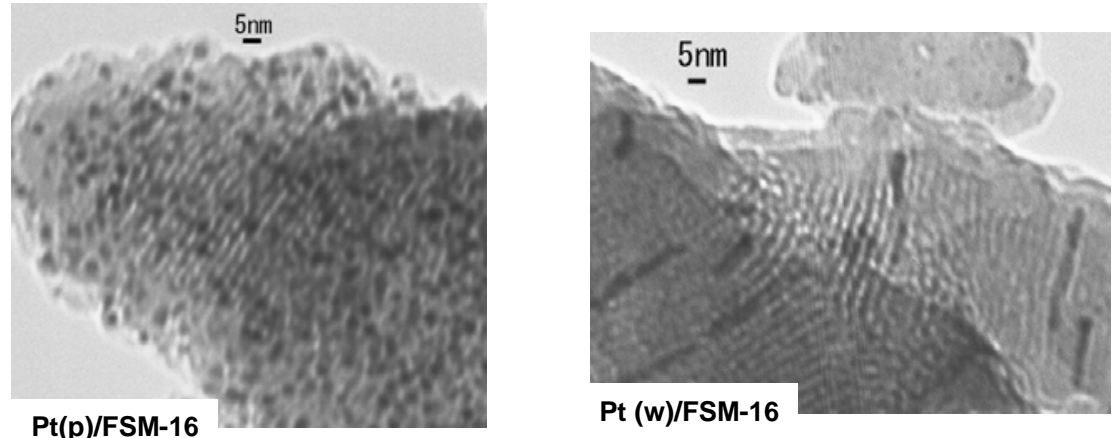

Figure S6. TEM images of the recovered catalysts after the PROX reactions. Conditions: CO 1\%, $\mathrm{O}_{2}$ $1 \%\left(\mathrm{O}_{2} / \mathrm{CO}=1\right), \mathrm{N}_{2} 5 \%, \mathrm{H}_{2}$ balance, SV $12000 \mathrm{ml} \mathrm{g}^{-1} \mathrm{~h}^{-1}, 0.1 \mathrm{MPa}, 353 \mathrm{~K}$.
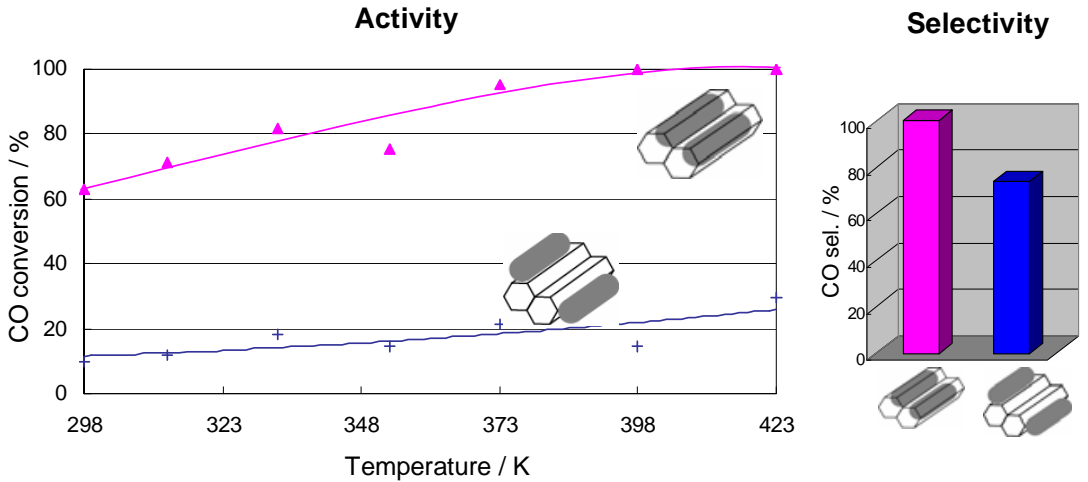

Figure S7. PROX of CO over Pt wires on internal and external surfaces of FSM-16. Conditions: CO $1 \%, \mathrm{O}_{2} 1 \%\left(\mathrm{O}_{2} / \mathrm{CO}=1\right), \mathrm{N}_{2}$ 5\%, $\mathrm{H}_{2}$ balance, SV $12000 \mathrm{ml} \mathrm{g}^{-1} \mathrm{~h}^{-1}, 0.1 \mathrm{MPa}, 298-423 \mathrm{~K}$. 

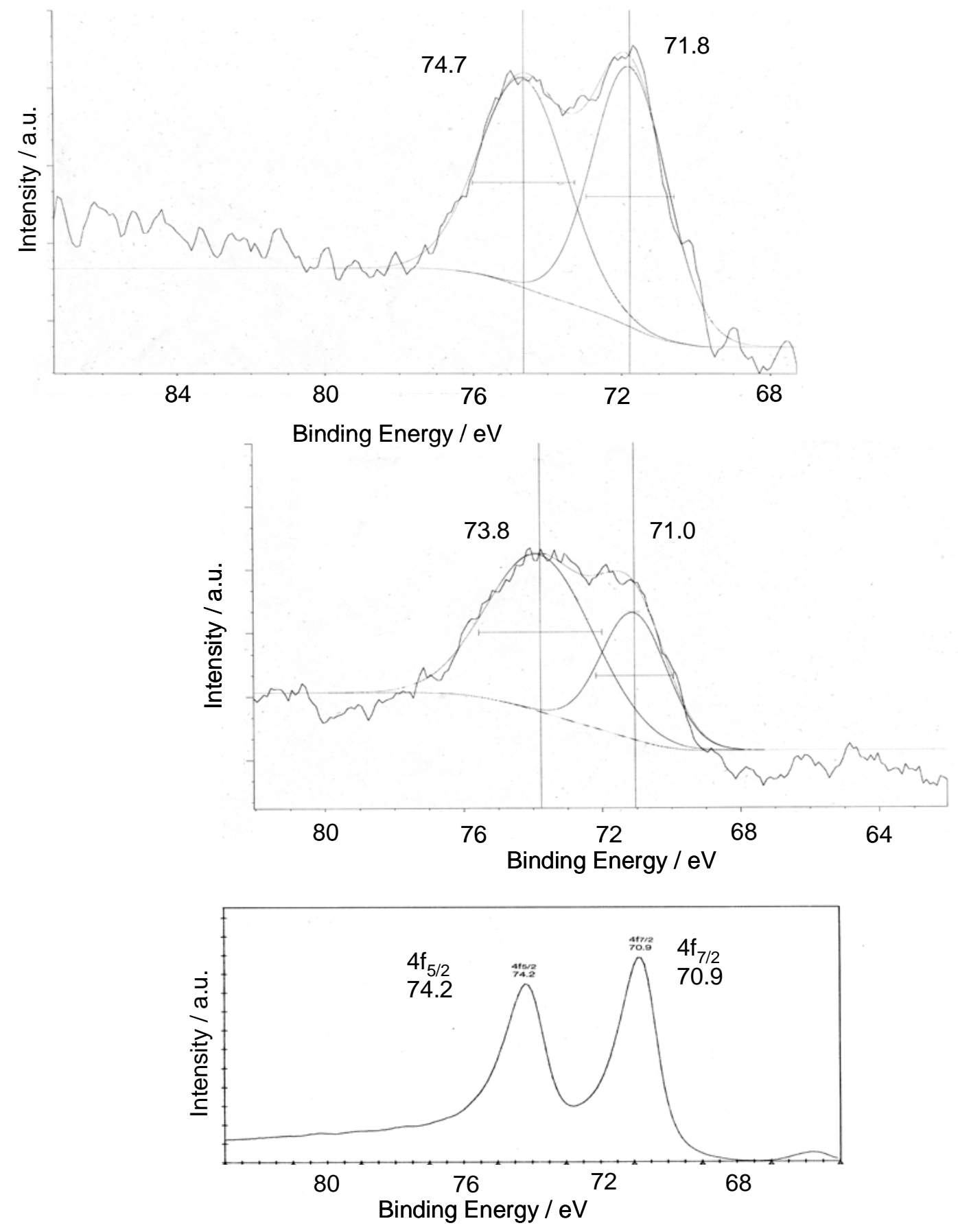

Figure S8. XPS of (a) Pt(p)/HMM-1, (b) Pt(p)/FSM-16 and (c) Pt foil. 


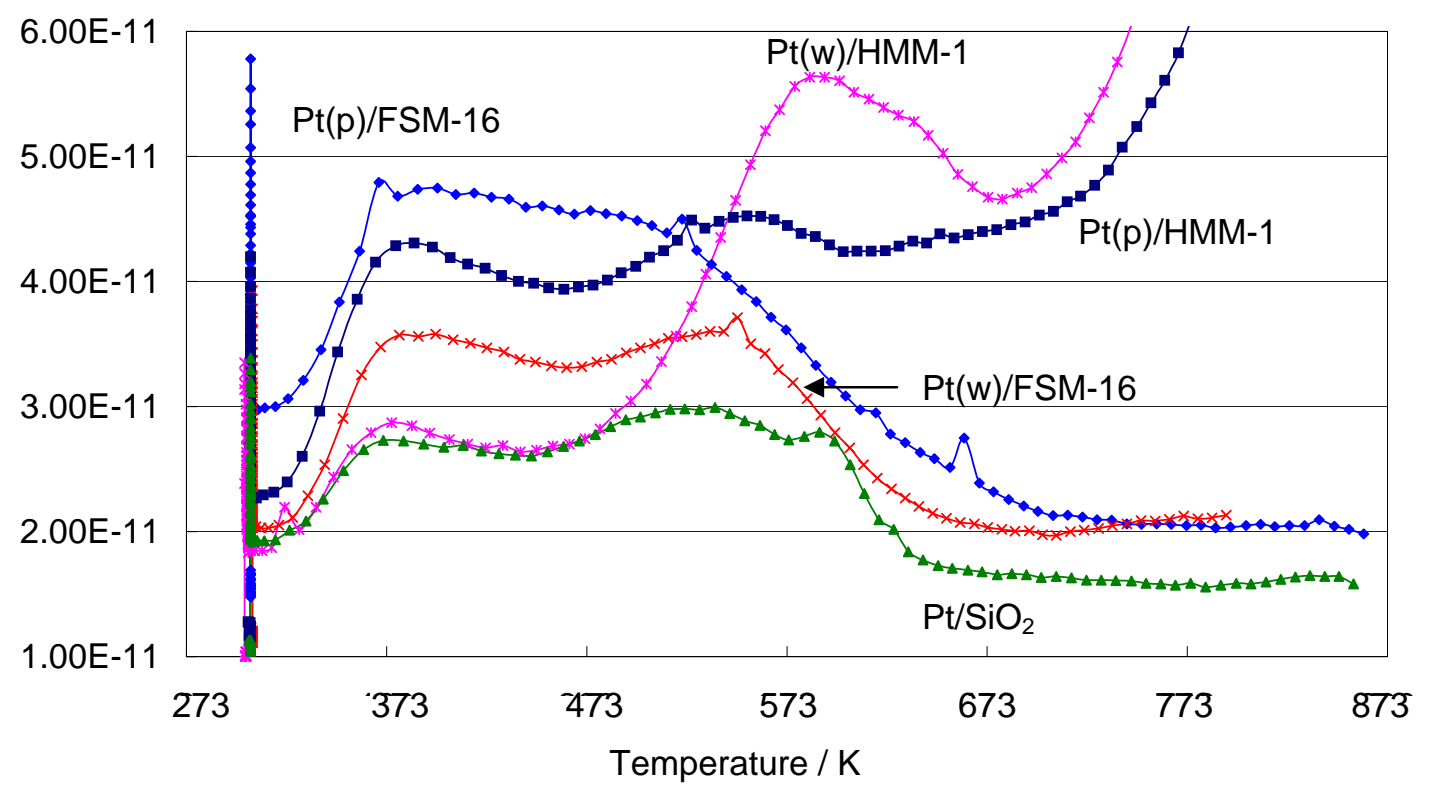

Figure S9. TPD profiles of $\mathrm{CO}(\mathrm{m} / \mathrm{e}=28)$ for supported Pt catalysts in flowing He. Peaks above $673 \mathrm{~K}$ for $\mathrm{Pt}(\mathrm{p}) / \mathrm{HMM}-1$ and $\mathrm{Pt}(\mathrm{w}) / \mathrm{HMM}-1$ are due to $\mathrm{CH}_{2} \mathrm{CH}_{2}(\mathrm{~m} / \mathrm{e}=28)$ formed by the decomposition of $\mathrm{O}_{3} \mathrm{Si}-\mathrm{CH}_{2} \mathrm{CH}_{2}-\mathrm{SiO}_{3}$ - units in HMM-1.

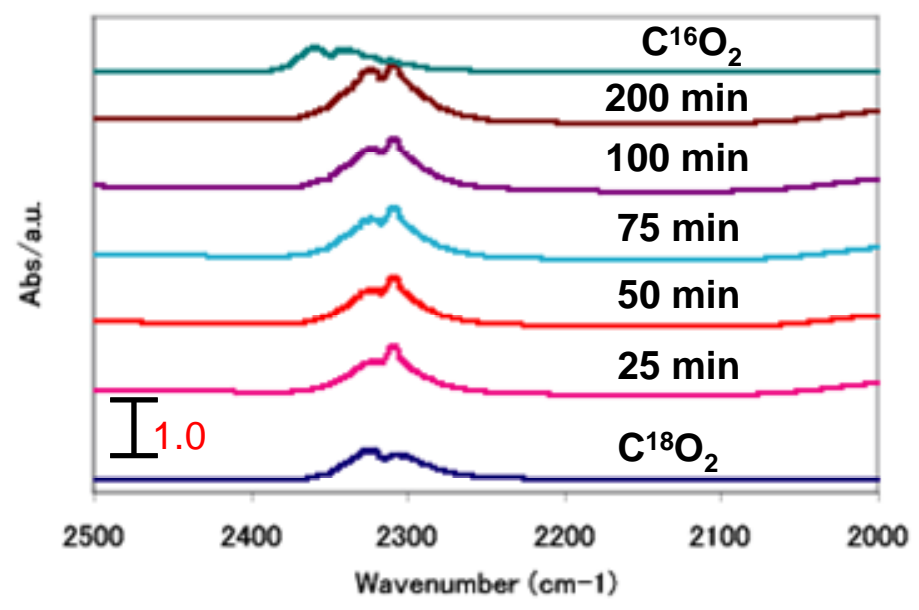

Figure S10. IR spectra after admission of $\mathrm{C}^{18} \mathrm{O}_{2}$ (30 Torr) to Pt(p)/FSM-16 at $293 \mathrm{~K}$. 